\title{
On the impact of control channel reliability on coordinated multi-point transmission
}

\author{
Zoltán Mayer ${ }^{*}$, Jingya Li, Agisilaos Papadogiannis and Tommy Svensson
}

\begin{abstract}
In the heterogeneous networks (HetNets), co-channel interference is a serious problem. Coordinated multi-point (COMP) transmission has emerged as a powerful technique to mitigate co-channel interference. However, all CoMP techniques rely on information exchange through reliable control channels, which are unlikely to be available in HetNets. In this paper, we study the effect of unreliable control channels, consisting of the access links and backhaul links, on the performance of COMP. A control channel model is introduced by assigning link failure probability (LFP) to backhaul and access links for the cooperative clusters. Three CoMP architectures, namely the centralized, semi-distributed, and fully distributed are analyzed. We investigate the probability of deficient control channels reducing quality of service and impeding transmission. General closed form expressions are derived for the probability of a cooperative transmission node staying silent in a resource slot due to unreliable control links. By evaluating the average sum rate of users within a CoMP cluster, we show that the performance gains offered by CoMP quickly diminish, as the unreliability of the control links grows.
\end{abstract}

Keywords: Coordinated multi-point (CoMP); Heterogeneous networks (HetNet); Backhaul reliability; Control channel; Link failure probability

\section{Introduction}

Driven by the increasing popularity of connected devices in wireless communication systems, e.g., smartphones and tablets, mobile broadband traffic is growing rapidly. As cloud-based services become essential to our daily lives, users want to be connected anytime and from anywhere [1]. Traditional macrocell systems fall short to satisfy these needs, partly because increasing the available frequency spectrum is not an option, due to the fact that bandwidth is an extremely expensive and increasingly scarce commodity which is severely regulated. Macrocells can be also inadequate in providing indoor coverage due to the signal attenuation while penetrating the outer walls of the buildings [2]. More importantly, since numerous users are in the coverage area of each macrocell, any single user equipment (UE) gets only a small share of network resources, limiting throughput. To satisfy demand for high data rates while reducing cost per bit, the spectral efficiency of cellular networks needs to be significantly increased [3].

*Correspondence: zoltan.mayer@alumni.chalmers.se

Department of Signals and Systems, Chalmers University of Technology, Gothenburg SE-412 96, Sweden
The spectral efficiency of a cellular network can be improved by increasing the cell density and reducing the transmission power of the network nodes. Hence, embedding low-power nodes into the existing networks, so as to form a heterogeneous network (HetNet), has emerged as a viable way to increase network capacity [4]. Short-range, plug-and-play indoor base stations promise to boost achievable throughput and fill the coverage holes. However, a major challenge of HetNets is the management of co-channel interference [5]. From information theory, it is known that inter-cell interference can be overcome, if transmission nodes (TN) process signals in a cooperative manner [6]. Recently, such techniques are referred to as coordinated multi-point (CoMP) [7-9]. CoMP schemes allow interference mitigation through joint and coherent transmission from multiple TNs, but at the cost of increased complexity and signaling overhead [10-12].

In spite of the significant performance gain that CoMP can provide, the use of CoMP in real systems results in a substantial control signaling overhead. The control signaling information includes the channel state information (CSI) sent over the access links between TNs and users, as well as the user scheduling and transition decisions sent

\section{是 Springer}

(c) 2014 Mayer et al: licensee Springer. This is an Open Access article distributed under the terms of the Creative Commons Attribution License (http://creativecommons.org/licenses/by/2.0), which permits unrestricted use, distribution, and reproduction in any medium, provided the original work is properly cited. 
over backhaul links between different $\mathrm{TNs}^{\mathrm{a}}$. In this paper, these access links and backhaul links are named as control channels. Despite the importance of backhaul unreliability, its impact on multi-node cooperative systems has only been partially investigated in relaying networks [13-15]. In [16], the impact of heterogeneous backhauls is investigated for coherent CoMP transmission in the downlink of femtocells, assuming that both the wired and wireless backhaul links are reliable. To the best of our knowledge, this work, partly included in [17], is the first one that analyzes the impact of control channel unreliability in CoMP systems. In addition, in this work, different transmission schemes are investigated under different CoMP network architectures [18-21], considering unreliable control channels.

The efficiency of all CoMP schemes rely heavily on the properties of the control channels. Traditionally, backhaul links are assumed to be highly reliable, which are less likely to be available in the heterogeneous and future dense networks. This is because the high number of access nodes would need to be accompanied by a proportionally high financial investment in order to build high quality wireline backhaul [22]. Furthermore, the topology of heterogeneous access points, i.e., some will be mounted on high towers (macro stations), others will be deployed on the street level below rooftops (pico and relay stations) and others will be indoors (femtocells), suggests that backhaul links interconnecting access nodes are wireless and without guaranteed line of sight [23].

This paper aims to extend and further develop the work originally reported by the authors in [17]. Motivated by the HetNet scenario, we evaluate the downlink of a cooperative wireless network and study the impact of control channel reliability on the system performance with different CoMP techniques. A control channel model is introduced for the cooperative systems under different network architectures. In addition to the work reported in [17], the authors also investigated the fully distributed network architecture. The model of the semi-distributed architecture has also been modified to provide a fairer comparison. An analytical approach is taken to investigate how backhaul and access link reliability affects the operation of the cooperating TNs. We provide general closed form expressions to assess the probability of a TN staying silent in a resource slot, depending on the link failure probabilities (LFP) of the backhaul network and the access links. Under each considered network CoMP architecture, the zero-forcing coherent joint transmission scheme and the multi-point coordinated scheduling scheme are studied and compared. We have found that although higher rates are achievable with both coordinated scheduling and coherent joint transmission, both schemes are very sensitive to control channel reliability. The semi- and fully distributed architectures are more robust to LFP, as the performance of the CoMP schemes under these architectures will converge to traditional single cell transmission, as LFP grows.

The rest of the paper is organized as follows. In Section 2, we present the signal and system model. In Section 3, the examined control channel models for different system architectures are introduced. Section 4 illustrates how backhaul and access link reliability affects TN operation under the described CoMP architectures. The numerical results are discussed in Section 5, and the conclusions are drawn in Section 6.

Notation: here, ()$^{H},()^{T},()^{-1}$, and ()$^{+}$denote the conjugate transpose, transpose, matrix inversion, and matrix pseudo-inversion operations, respectively. The notations $\mathbf{1}_{[m \times n]}$ and $\mathbf{0}_{[m \times n]}$ represent the matrix with $m$ rows and $n$ columns filled with ones and zeros, respectively. $\mathbf{X}(n,:)$ denotes the $n$th row of matrix $\mathbf{X}$. $\mathbf{N}$ refers to the set of natural numbers. $|\mathcal{M}|$ denotes the cardinality of the set $\mathcal{M}$. $\odot$ represents the element-wise multiplication.

\section{Signal and system model}

In this paper, we consider the downlink of a cooperative system, consisting of $N$ single-antenna TNs and $M$ single-antenna UEs. The UEs are grouped together using a particular resource slot. In the following, we assume $M=N$ and the serving TN of UE $m=n$ is TN $n$. The $N$ TNs are assumed to have the same maximum power constraint $P_{\max }$ and to share the same resource slot. Let $\mathbf{x}=\left[x_{1}, \ldots, x_{N}\right]^{T}$ denote the signal vector transmitted from all $N$ TNs, with $x_{n}^{H} x_{n} \leq P_{\max }$ for all $n \in\{1, \ldots, N\}$. The received signal at UE $m$ can then be expressed as

$$
y_{m}=\mathbf{h}_{m} \mathbf{x}+n_{m},
$$

where $\mathbf{h}_{m}=\left[h_{m 1}, \ldots, h_{m N}\right]$ denotes the channel state vector between UE $m$ and all $N$ TNs. Here, $n_{m}$ is the sum of the thermal noise and the uncoordinated out-of-cluster interference, modeled as independent complex additive white Gaussian noise [24].

Each UE $m$ estimates its channel state vector $\mathbf{h}_{m}$ and feeds it back to its serving TN $m$ via the access link. We assume that the UEs use orthogonal resource slots during CSI feedback; therefore, the outage probability of these uplink control channels can be evaluated in terms of the minimum signal-to-noise ratio, $\rho_{0}$, that is required for successful transmission. The control unit (CU) gathers CSI from the cooperating TNs via backhaul links and designs the transmission parameters [25]. It is assumed that the CSI of all UEs within the system, named as full CSI, is corrupted via control channels, i.e., the backhaul and access links. Hence, the system channel matrix available at the CU is denoted as $\hat{\mathbf{H}}=\left[\hat{\mathbf{h}}_{1}^{T}, . ., \hat{\mathbf{h}}_{M}^{T}\right]^{T} \in \mathcal{C}^{M \times N}$, which will be used for the scheduling and transmission scheme design. 


\subsection{Coherent joint transmission}

Assume that the data symbols of all the $M$ UEs within the cluster are shared among the $N$ coordinated TNs. A linear precoding approach, zero-forcing, is considered as the coherent joint transmission scheme in this section. Note that with linear precoding among $N$ single-antenna TNs, at most $N$ single-antenna UEs can be served on the same resource slot without inter-user interference. Let $\mathcal{M}$ denote the set of scheduled UEs in a given resource slot, with $\mathcal{M} \subseteq\{1, \ldots, M\}$ and $|\mathcal{M}| \leq N$. Let $\mathbf{b} \in \mathbf{C}^{|\mathcal{M}|}$ be the data symbols of the selected UEs in set $\mathcal{M}$. A precoding matrix $\mathbf{W}=\left[\mathbf{w}_{1}, \ldots, \mathbf{w}_{|\mathcal{M}|}\right] \in \mathcal{C}^{N \times|\mathcal{M}|}$ is designed for mapping the data symbol vector $\mathbf{b}$ into the transmit signal vector $\mathbf{x}$, that is,

$$
\mathbf{x}=\mathbf{W b} \text {. }
$$

The $m$ th column of $\mathbf{W}, \mathbf{w}_{m}=\left[w_{1 m}, \ldots, w_{N m}\right]^{T}$, is the precoding vector for UE $m$ in the set $\mathcal{M}$. The received signal of UE $m$ can be rewritten as

$$
y_{m}=\mathbf{h}_{m} \mathbf{w}_{m} b_{m}+\sum_{i \in \mathcal{M}, i \neq m} \mathbf{h}_{m} \mathbf{w}_{i} b_{i}+n_{m} .
$$

Let $p_{m}=b_{m} b_{m}^{H}$ denote the symbol power allocated to UE $m$ across the $N$ TNs. The signal-to-interference-plusnoise ratio (SINR) of UE $m$ is then given by

$$
\rho_{m}=\frac{\left\|\mathbf{h}_{m} \mathbf{w}_{m}\right\|^{2} p_{m}}{\sum_{i \in \mathcal{M}, i \neq m}\left\|\mathbf{h}_{m} \mathbf{w}_{i}\right\|^{2} p_{i}+\sigma^{2}} .
$$

Thus, the sum rate of the cluster can be expressed as

$$
C=\sum_{m \in \mathcal{M}} \log _{2}\left(1+\rho_{m}\right)
$$

Using zero-forcing precoding, the precoding matrix, $\mathbf{W}$, is obtained as the pseudo-inverse of the channel matrix, $\hat{\mathbf{H}}$, available at the $\mathrm{CU}$, that is,

$$
\mathbf{W}=\hat{\mathbf{H}}^{-1} \text {. }
$$

In order to reduce the complexity, a sub-optimal equal power allocation is considered [11]. As a first step, $\mathbf{W}$ is normalized column-wise, then for any given UE set, $\mathcal{M}$, the power allocation vector is derived as

$$
\mathbf{p}=\left\{\min _{n=1, \ldots N} \frac{P_{\max }}{\sum_{m \in \mathcal{M}}\left\|w_{n m}\right\|^{2}}\right\} \mathbf{1}_{[|\mathcal{M}| \times 1]} .
$$

By solving the joint power allocation of Equation 7 for every possible UE set $\mathcal{M}$, the chosen UE set $\mathcal{M}^{\mathrm{JT}}$ and $\mathbf{p}^{\mathrm{JT}}$ will be the ones that achieve the highest $\sum_{m=1}^{M} \log _{2}$ $\left(1+\hat{\rho}_{m}\right)$, where $\hat{\rho}_{m}$ is derived from Equation 4 by using the obtained $\hat{\mathbf{h}}_{m}$ at the CU instead of the true channel vector $\mathbf{h}_{m}$. In the following, this zero-forcing coherent joint transmission scheme is denoted as JT.

\subsection{Coordinated scheduling}

In the considered coordinated scheduling scheme, data to a single UE is only transmitted from its serving TN, which is selected based on the long-term channel quality measurements, including path loss and shadow fading. Hence, user data exchange between TNs is not needed. It is assumed that a TN can transmit data to at most one UE in any given resource slot.

Let $P_{m}=b_{m}^{H} b_{m}$ denote the transmit power of TN $m$ to UE $m$, with $P_{m} \leq P_{\max }$. Then, the SINR for UE $m$ is given as

$$
\rho_{m}=\frac{\left\|h_{m m}\right\|^{2} P_{m}}{\sum_{j=1, j \neq m}^{j=N}\left\|h_{m j}\right\|^{2} P_{j}+\sigma^{2}}
$$

Thus, the sum rate can be calculated by Equation 5 .

UE scheduling and power allocation decisions are jointly made at the CU to control ICI. With the gathered channel matrix, $\hat{\mathbf{H}}$, the CU designs the UE selection indicator matrix $\mathbf{S}$ and the power allocation vector $\mathbf{P}=$ $\left[P_{1}, \ldots, P_{n}\right]$, in order to maximize the sum rate subject to per-TN power constraints. Based on [26] and [27], a suboptimal but efficient binary power control (BPC) is considered for this coordinated scheduling scheme, i.e., $P_{n}=0$ or $P_{\max }$ for $\forall n \in\{1, \ldots, N\}$. Then, the relaxed problem becomes an exhaustive binary search. The CU searches all feasible boundary point sets, i.e., $P_{n}=0$ or $P_{\max }$ for $\forall n \in\{1, \ldots, N\}$. The chosen transmit power vector $\mathbf{P}^{C S}$ will be the one that achieves the highest $\sum_{m=1}^{M} \log _{2}\left(1+\hat{\rho}_{m}\right)$, where $\hat{\rho}_{m}$ is derived from Equation 8 by using the obtained $\hat{\mathbf{h}}_{m}$. In this paper, this scheme is named as CS.

\section{Control channel models}

In this section, we introduce the control channel models considered for single cell transmission and for the cooperative systems under different network architectures, i.e., the centralized, semi-distributed, and fully distributed CoMP architectures. We assume that each TN $n$ is linked to one user, i.e., UE $n$, yet each $\mathrm{TN} n$ is potentially serving other UEs in the cluster. Here, we leave the LFP model of each control link unspecified to allow general control channel models for different network architectures. A specific control channel LFP model is presented in Section 4.

\subsection{Single cell transmission}

Single cell transmission without $\mathrm{TN}$ coordination (Figure 1), denoted as SC, is used as a baseline. For SC transmission, the data blocks sent from the core network to TN $n$ will only contain the data symbol for UE $n$. All TNs will always be transmitting, if user data is available, 


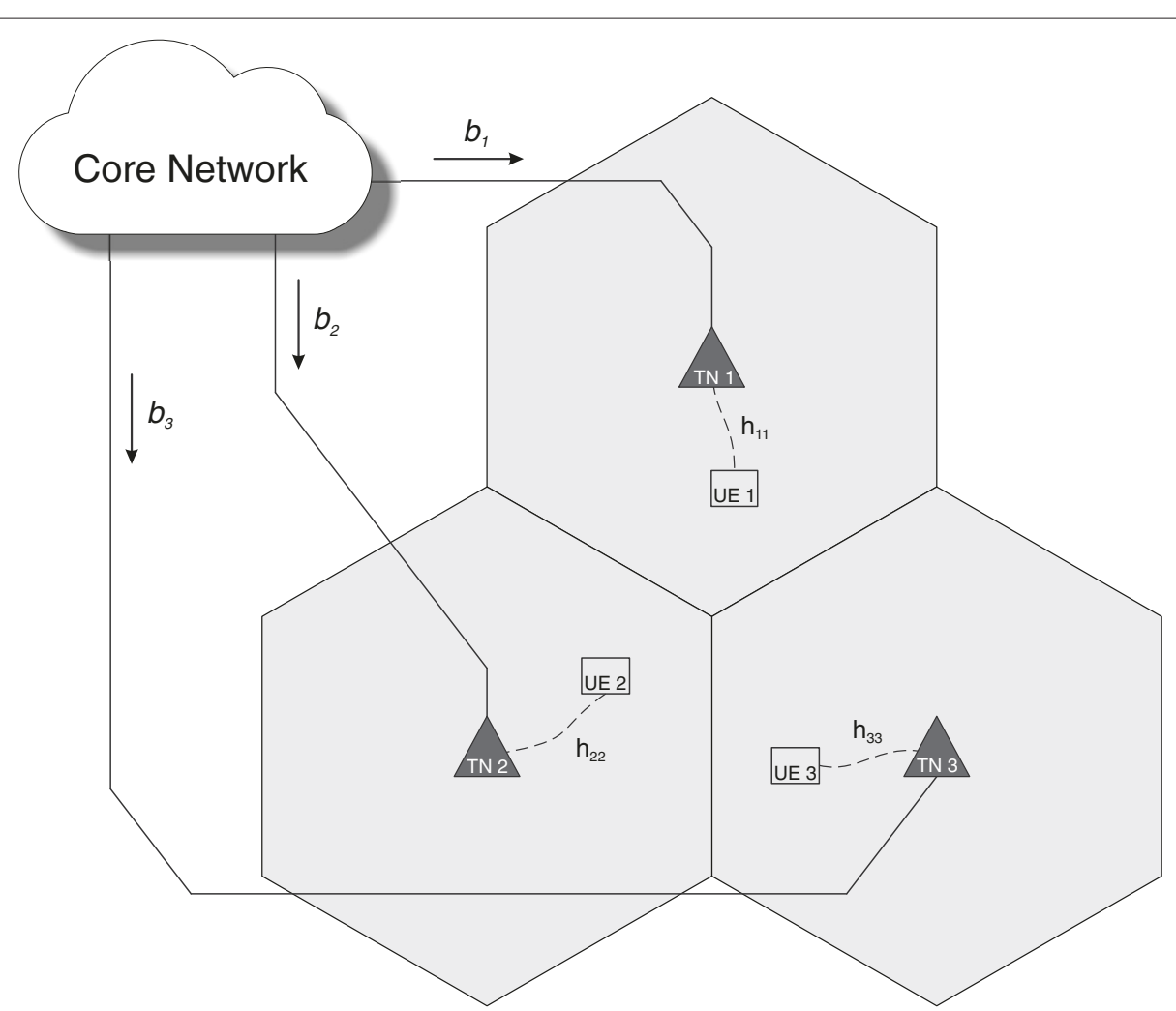

Figure 1 Single cell transmission. Traditional single cell transmission, with no cooperation between the TNs, is used as a baseline for performance evaluation.

even if the channel conditions are poor over the access link. There is no cooperation between TNs; therefore CSI needs not to be shared.

\subsection{Centralized CoMP architecture}

The centralized architecture, introduced in [24] and [28], is depicted in Figure 2. Under this architecture, each UE $n$ feeds back its CSI to its serving TN $n$ via access link in the first step. Next, the TNs forward their received local CSI to the CU via backhaul links. Based on the gathered $\hat{\mathbf{H}}$, the $\mathrm{CU}$ constructs the precoding matrix for the JT scheme or makes scheduling decisions for the CS scheme. Once the decisions are made, the $\mathrm{CU}$ forwards them via backhaul links to each coordinated TN. Hence, backhaul links are used twice, i.e., gathering full CSI and distributing transmission decisions. We assume that the user data distributed from the core network to the $\mathrm{CU}$ is fully reliable.

All control channels are modeled to be prone to errors, leading to losing partial CSI of the system at the $\mathrm{CU}$ and/or losing precoded user data at the cooperating TNs. The full CSI available at the CU is affected by the LFPs of the access links between each UE $n$ and its serving TN $n$, as well as the LFPs of the backhaul links between each TN $n$ and the CU. LFPs are modeled as independent binary discrete random variables. Hence, the available system channel matrix at $\mathrm{CU}, \hat{\mathbf{H}}$, is obtained as

$$
\hat{\mathbf{H}}=\mathbf{H} \odot \mathbf{H}^{\text {Access mask }} \odot \mathbf{H}^{\text {Backhaul mask }},
$$

where $\mathbf{H}$ is the perfect system channel matrix ${ }^{\mathrm{b}}$. Here, $\mathbf{H}^{\text {Access mask }}$ is a binary mask matrix, where the $n$th row vector, $\mathbf{H}^{\text {Access mask }}(n,:)$, is either $\mathbf{0}_{[1 \times N]}$ with probability $P_{n, n}^{\mathrm{O}}$ or $\mathbf{1}_{[1 \times N]} . P_{n, n}^{\mathrm{O}}$ is the LFP of the access link over which user $n$ feeds back the CSI to TN $n$. Also, $\mathbf{H}^{\text {Backhaul mask }}$ is a binary mask matrix, where each row vector, $\mathbf{H}^{\text {Backhaul mask }}(n,:)$, is either $\mathbf{0}_{[1 \times N]}$ with probability $P_{\mathrm{F}_{n}}^{\mathrm{C}}$ or $\mathbf{1}_{[1 \times N]}$. Here, $P_{\mathrm{F}_{n}}^{\mathrm{C}}$ is the LFP of the backhaul link where the CSI is forwarded from TN $n$ to the CU. Note that the precoding matrix, $\mathbf{W}$, is obtained according to Equation 6, which is based on $\hat{\mathbf{H}}$. Hence, it is still possible to provide data transmission without having received all the CSI elements.

For the JT scheme, the user data distributed from the CU to TN $n, x_{n}$, contains the precoded data symbols for the scheduled UEs, i.e., $x_{n}=\mathbf{W}(n,:) \times \mathbf{b}=$ $\sum_{m \in \mathcal{M}} w_{n m} b_{m}$. Let $P_{\mathrm{F}_{n}}^{\mathrm{D}}$ denote the LFP of the backhaul links where the precoded data symbols are distributed from the CU to TN $n$. Similarly, to model the data loss via backhaul links to each TN, a binary mask matrix, 


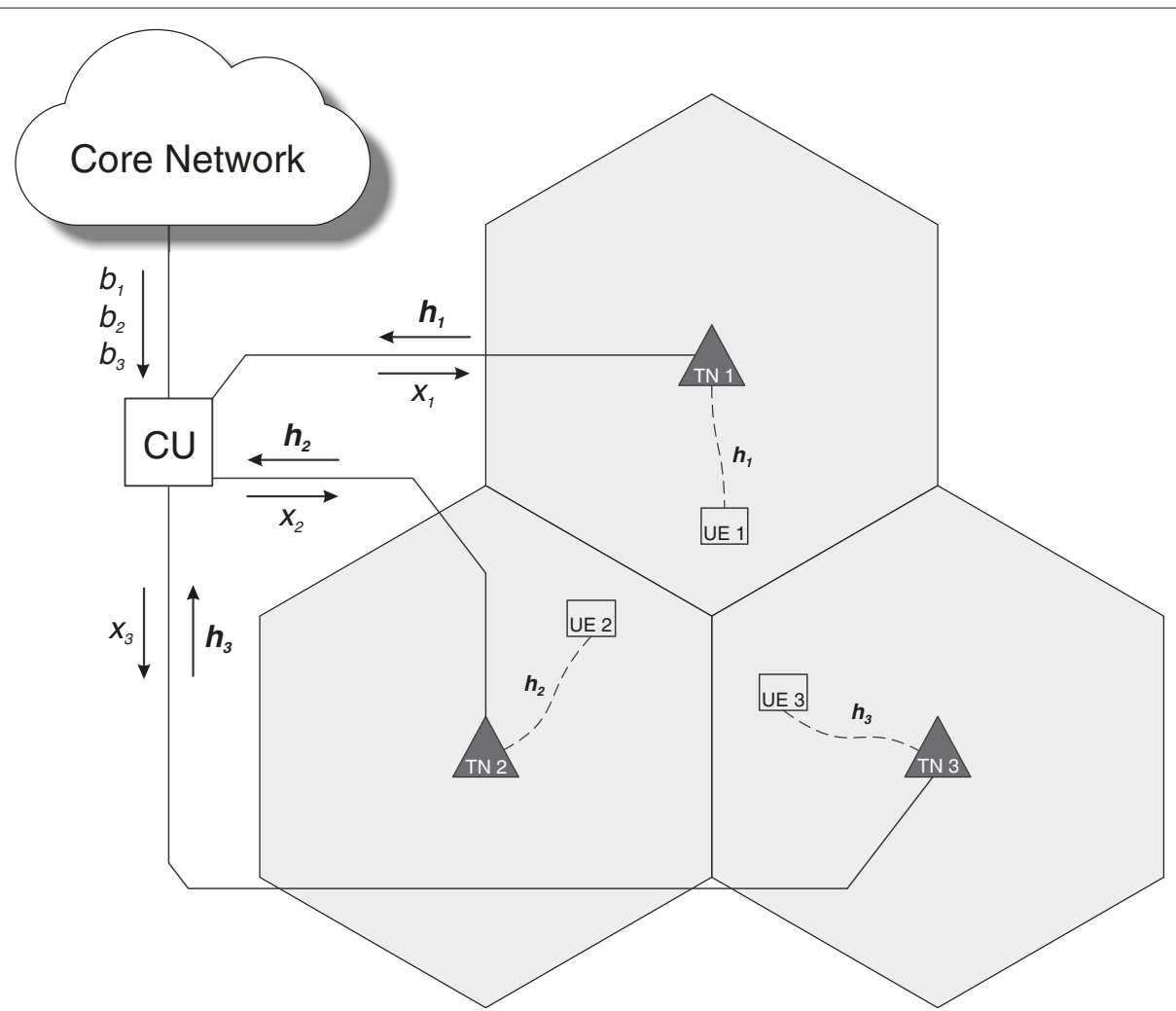

Figure 2 Centralized CoMP architecture. Under the centralized CoMP architecture, the TNs forward CSI to a control unit that applies precoding or makes scheduling decisions.

$\mathbf{W}^{\text {Backhaul mask }}$, is applied to the precoding matrix designed at the $\mathrm{CU}, \mathbf{W}$, as

$$
\hat{\mathbf{W}}=\mathbf{W} \odot \mathbf{W}^{\text {Backhaul mask }},
$$

which erases each row vector of $\mathbf{W}$ independently, with a probability of $P_{\mathrm{F}_{n}}^{\mathrm{D}}$. The SINR of the scheduled UEs can be derived by substituting $\hat{\mathbf{W}}$ into Equation 4; the sum rate can then be obtained from Equation 5.

Example 1. A cooperative cluster comprises of $N=3$ TNs, as shown in Figure 2. All UEs feed back the channel state vector to their serving TN. Then, each TN n forwards the received local channel state vector via backhaul links to the CU. Assume that there are link failures on the access link between UE 2 and TN 2 and the backhaul link from TN 3 to the CU. Then, according to Equation 9, the full CSI available at the $C U, \hat{\mathbf{H}}$, can be modeled as

$$
\hat{\mathbf{H}}=\left[\begin{array}{l}
\mathbf{h}_{1} \\
\mathbf{h}_{2} \\
\mathbf{h}_{3}
\end{array}\right] \odot\left[\begin{array}{lll}
1 & 1 & 1 \\
0 & 0 & 0 \\
1 & 1 & 1
\end{array}\right] \odot\left[\begin{array}{lll}
1 & 1 & 1 \\
1 & 1 & 1 \\
0 & 0 & 0
\end{array}\right] .
$$

Based on $\hat{\mathbf{H}}$, the precoding matrix $\mathbf{W}$ is designed at the CU. Considering a link failure on the backhaul link between the
CU and TN 1 when the precoded user data is distributed from the CU to all TNs, $\hat{\mathbf{W}}$ becomes

$$
\hat{\mathbf{W}}=\mathbf{W} \odot\left[\begin{array}{lll}
0 & 0 & 0 \\
1 & 1 & 1 \\
1 & 1 & 1
\end{array}\right]
$$

For the CS scheme, where data to a single UE is only transmitted from its serving $\mathrm{TN}$, the data loss due to backhaul unreliability is modeled as

$$
\hat{b}_{n}=b_{n} b_{n}^{\text {mask }}
$$

Here, $b_{n}^{\text {mask }}$ is a binary mask variable which erases the data symbol of UE $n$ with probability $P_{\mathrm{F}_{n}}^{\mathrm{D}}$. Thus, the SINR of each UE $n$ can be calculated by substituting $\mathbf{P}^{\text {mask }}$ into Equation 8 , where the $n$th element, $\mathbf{P}_{n}^{\text {mask }}$, is derived by $\mathbf{P}_{n}^{\text {mask }}=b_{n}^{\text {mask }} \mathbf{P}_{n}^{C S}$.

\subsection{Semi-distributed CoMP architecture}

The semi-distributed architecture, introduced in [29] and [30], is depicted in Figure 3. Under this architecture, each user feeds back the CSI vector to its serving TN. Then, the received local CSI vectors are shared between TNs via interconnecting backhaul links. Therefore, each TN $n$ receives $N-1$ non-local CSI vectors from $N-1$ 


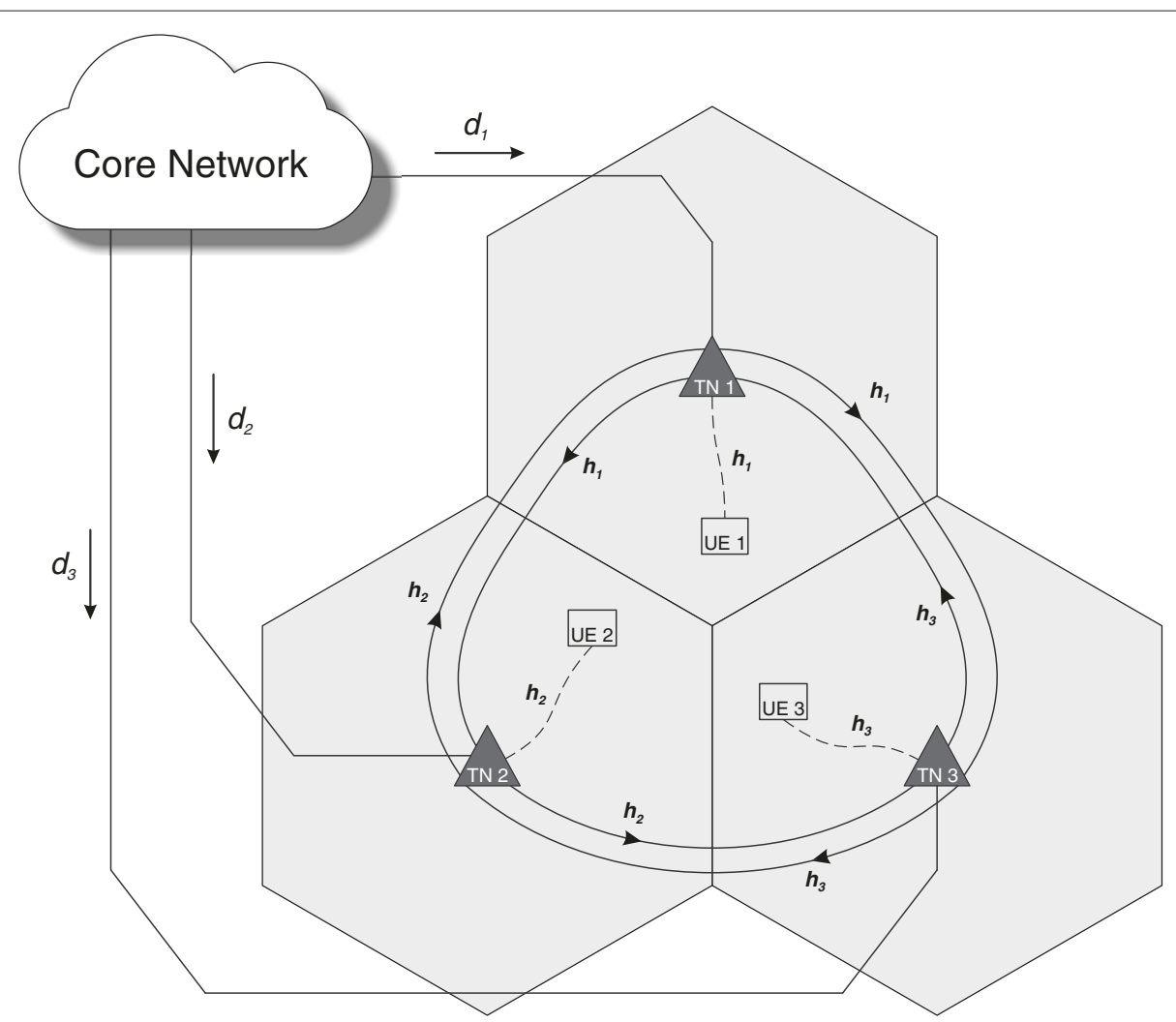

Figure 3 Semi-distributed CoMP architecture. Under the semi-distributed CoMP architecture, each TNs acts as a control unit; local CSI is shared between the cooperating TNs.

coordinated TNs via backhaul links, thus acquiring $\hat{\mathbf{H}}_{n}$, which can be modeled by

$$
\hat{\mathbf{H}}_{n}=\mathbf{H} \odot \mathbf{H}^{\text {Access mask }} \odot \mathbf{H}_{n}^{\text {Backhaul mask }},
$$

where $\mathbf{H}^{\text {Access mask }}$ is a binary mask matrix defined in Equation 9, modeling the link failure of the access links.

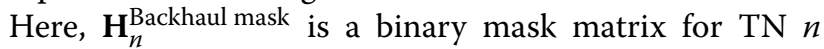
modeling the effect of backhaul link failures. Similarly to Equation 9, the $m$ th row vector, $\mathbf{H}_{n}^{\text {Backhaul mask }}(m,:)$, is either $\mathbf{0}_{[1 \times N]}$ with probability $P_{\mathrm{F}_{m, n}}$ or $\mathbf{1}_{[1 \times N]}$, with $m \neq n$. Note that the local CSI vector for each TN $n$, is directly fed back from its own UE $n$. Hence, the $n$th row of $\hat{\mathbf{H}}_{n}$ will not be affected by the LFP of backhaul links. Thus, the $n$th row of $\mathbf{H}_{n}^{\text {Backhaul mask }}=\mathbf{1}_{[1 \times N]}$ with probability 1 .

Assume that user data is safely received at each TN from the core network. Based on the gathered $\hat{\mathbf{H}}_{n}$, each cooperating $\mathrm{TN} n$ acts as a $\mathrm{CU}$, independently designing its own precoding weights and the power allocation vector for JT, or making scheduling decisions for CS. Transmission decisions are then locally applied to the user data at each TN.

For the JT scheme, the data blocks, sent from the core network to TN $n$, contain all data symbols for the scheduled UEs in the cluster, $d_{n}=\left[b_{1}, \ldots, b_{m}\right]$. Each TN $n$ independently designs the precoding matrix $\mathbf{W}_{n}$ based on the gathered system matrix $\hat{\mathbf{H}}_{n}$. The $n$th row of $\mathbf{W}_{n}$, i.e., $\mathbf{W}_{n}(n,:)$, is then chosen by TN $n$ as the precoding vector for mapping the user data symbols into the transmit signal. Therefore, the system precoding matrix will be

$$
\hat{\mathbf{W}}=\left[\begin{array}{c}
\mathbf{W}_{1}(1,:) \\
\mathbf{W}_{2}(2,:) \\
\vdots \\
\mathbf{W}_{N}(N,:)
\end{array}\right] .
$$

The SINR of the scheduled UEs can be derived by substituting $\hat{\mathbf{W}}$ into Equation 4. Finally, the sum rate can then be obtained from Equation 5 .

Example 2. A cooperative cluster comprises of $N=3$ TNs, as shown in Figure 3. Consider modeling the gathered $\hat{\mathbf{H}}_{1}$ at TN 1. Firstly, all UEs feed back the local channel state vector to their serving TN. Hence, TN 1 receives the channel state vector $\mathbf{h}_{1}$ from UE 1, through the access link. Then, TN 2 and TN 3 share $\mathbf{h}_{2}$ and $\mathbf{h}_{3}$ with TN 1 through unreliable backhaul links. Assume that there are link failures on the access link between UE 2 and TN 2 and on the 
backhaul link between TN 3 to TN 1. Then, according to Equation 12, $\hat{\mathbf{H}}_{1}$ can be modeled as

$$
\hat{\mathbf{H}}_{1}=\left[\begin{array}{l}
\mathbf{h}_{1} \\
\mathbf{h}_{2} \\
\mathbf{h}_{3}
\end{array}\right] \odot\left[\begin{array}{lll}
1 & 1 & 1 \\
0 & 0 & 0 \\
1 & 1 & 1
\end{array}\right] \odot\left[\begin{array}{lll}
1 & 1 & 1 \\
1 & 1 & 1 \\
0 & 0 & 0
\end{array}\right] .
$$

Based on $\hat{\mathbf{H}}_{1}, T N 1$ designs the precoding matrix $\mathbf{W}_{1}$. Then, the first row of $\mathbf{W}_{1}$, i.e., $\mathbf{W}_{1}(1,:)$, will be chosen as the precoding vector for $T N 1$. The system precoding matrix $\hat{\mathbf{W}}$ is then derived from Equation 13.

For the CS scheme, the downlink data block, $d_{n}$, which is distributed from the core network to each $\mathrm{TN} n$, contains only the data symbol of its own UE. Thus, $d_{n}=b_{n}$. Based on available $\hat{\mathbf{H}}_{n}$, each TN $n$ designs the transmit power vector $\mathbf{P}_{n}^{C S}$. The $n$th element of $\mathbf{P}_{n}^{\text {CS }}$, i.e., $\mathbf{P}_{n}^{\mathrm{CS}}(n)$ is then chosen by TN $n$ as the transmit power for UE $n$. Thus, the system transmit power vector will be

$$
\hat{\mathbf{P}}^{\mathrm{CS}}=\left[\mathbf{P}_{n}^{\mathrm{CS}}(1), \mathbf{P}_{n}^{\mathrm{CS}}(2), \ldots, \mathbf{P}_{N}^{C S}(N)\right] .
$$

The SINR of the scheduled UEs can be derived by substituting $\hat{\mathbf{P}}^{\mathrm{CS}}$ into Equation 8.

\subsection{Fully distributed CoMP architecture}

The fully distributed architecture, introduced in [25] and [31], is depicted in Figure 4. This architecture differs from the semi-distributed architecture (Section 3.3) in the way TN $n$ acquires the full CSI matrix $\hat{\mathbf{H}}_{n}$. All UEs in the cluster broadcast their local CSI vectors to all TNs, therefore, any row of the local gathered system channel matrix, $\mathbf{h}_{m}$, can be lost due to the LFP of the access links between UE $m$ and TN $n$. Hence, $\hat{\mathbf{H}}_{n}$ can be modeled by

$$
\hat{\mathbf{H}}_{n}=\mathbf{H} \odot \mathbf{H}_{n}^{\text {Access mask }},
$$

where $\mathbf{H}_{n}^{\text {Access mask }}$ is a binary mask matrix for TN $n$ modeling the link failures of the access links between TN $n$ and all UEs. ${ }^{c}$ Here, $P_{n, m}^{\mathrm{O}}$ is the outage probability between UE $m$ and TN $n$. Similarly to Equation 9, the $m$ th row vector, $\mathbf{H}_{n}^{\text {Access mask }}(m,:)$, is either $\mathbf{0}_{[1 \times N]}$ with probability $P_{n, m}^{\mathrm{O}}$ or $\mathbf{1}_{[1 \times N]}$, with $n \neq m$.

Note that in practice, the control information might be a quantized vector of the channel state information or a precoding matrix index that corresponds to the preferred codebook. In this case, the masking concept is still valid. Instead of using a masking matrix for the explicit channel matrix $\mathbf{H}$ or $\mathbf{W}$, a masking vector can be performed on the quantized vectors or the indices for modeling the control channel unreliability.

\section{Control channel reliability analysis}

In this section, we analyze the probability of a certain TN $n$ staying silent in a resource slot, $P_{n}^{\mathrm{S}}$, due to unreliable control channels. This may cause some UEs unserved or, as a worst-case scenario, impede all transmission with probability

$$
P^{\mathrm{W}}=\prod_{n=1}^{N} P_{n}^{\mathrm{S}} .
$$

The LFP of the control channels can be evaluated in terms of outage probability of the wireless channels. It is assumed that the UEs use orthogonal control channels for CSI feedback; therefore, outage probability is the probability that the instantaneous signal-tonoise ratio (SNR), $x$, is below that required for adequate reception [32]. The short-term variability of mobile radio signals can usually be described statistically with enough accuracy to be useful in mobile radio system analysis. If the desired SNR has a probability density function, $p_{\gamma}(z)$, then the probability that adequate reception will not be achieved, $P_{\text {out }}$, is

$$
P_{\text {out }}(x)=\operatorname{Pr}(\gamma<x)=\int_{0}^{x} p_{\gamma}(z) d z,
$$

where $\operatorname{Pr}($.$) denotes probability.$

In this section, we also provide closed form equations for $P_{n}^{\mathrm{S}}$ for different transmission schemes under different architectures, when the control channels are modeled with Rayleigh fading and a distance dependent path loss ${ }^{\mathrm{d}}$. Under Rayleigh fading, the received signal power is exponentially distributed, and the variations of the instantaneous SNR also follow an exponential distribution. Therefore, the outage probability can be obtained from

$$
P_{\text {out }}(x)=1-\exp \left(-\frac{x}{\Omega(d)}\right),
$$

where $x$ is the minimum required SNR for adequate reception, and $\Omega(d)$ is the mean SNR level at distance $d$ from the transmitter. Although more sophisticated channel models provide better statistical description, we use Rayleigh fading to maintain simplicity and tractability.

\subsection{Single cell transmission}

As discussed in 3.1, a certain TN $n$ will always be transmitting, regardless of the reliability of the control channels. Therefore, $P_{n}^{\mathrm{S}}$ can be expressed as

$$
P_{n}^{S}=0 \text {. }
$$

\subsection{Centralized CoMP architecture \\ 4.2.1 Coherent joint transmission}

Considering the JT scheme under the centralized architecture, based on the control channel model described in Section 3.2, TN $n$ will stay silent if 


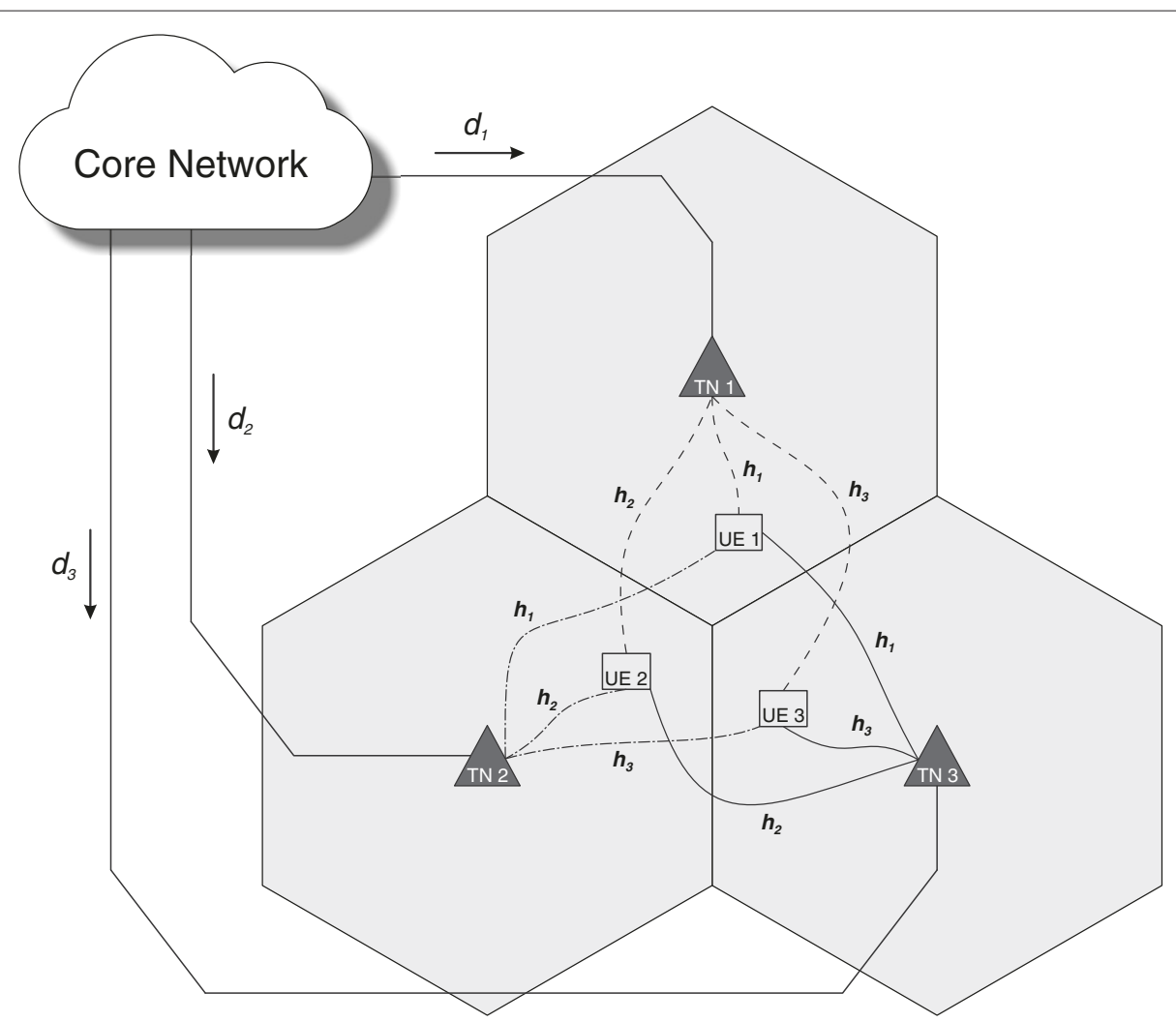

Figure 4 Fully distributed CoMP architecture. Under the fully distributed CoMP architecture, each TNs acts as a control unit; all CSI is obtained directly from the UEs by broadcast.

Case 1. All CSI vectors are lost at the CU so that no transmission decisions will be distributed from the CU to all TNs, otherwise,

Case 2. The precoded user data distributed from the $\mathrm{CU}$ to TN $n$ is lost. The probability that Case 1 happens is $\prod_{n=1}^{N}\left[P_{\mathrm{F}_{n}}^{\mathrm{C}}+\left(1-P_{\mathrm{F}_{n}}^{\mathrm{C}}\right) \cdot P_{n, n}^{\mathrm{O}}\right]$, where $P_{\mathrm{F}_{n}}^{\mathrm{C}}$ is the LFP of the backhaul link between TN $n$ and the $\mathrm{CU}$ when TN $n$ forwards the local CSI to the CU, and $P_{n, n}^{\mathrm{O}}$ is the LFP of the access link between UE $n$ and TN $n$, when UE $n$ feeds back the CSI to TN $n$. Case 2 happens with probability $P_{\mathrm{F}_{n}}^{\mathrm{D}}$, where $P_{\mathrm{F}_{n}}^{\mathrm{D}}$ is the LFP between the CU and TN n, when the CU distributes precoded user data to TN n (see Figure 5 for corresponding illustration). Therefore, the probability of TN $n$ staying silent, $P_{n}^{\mathrm{S}}$, for the JT scheme under the centralized architecture can be expressed as

$$
P_{n}^{\mathrm{S}}=P_{\mathrm{F}_{n}}^{\mathrm{D}}+\left(1-P_{\mathrm{F}_{n}}^{\mathrm{D}}\right) \prod_{n=1}^{N}\left[P_{\mathrm{F}_{n}}^{\mathrm{C}}+\left(1-P_{\mathrm{F}_{n}}^{\mathrm{C}}\right) \cdot P_{n, n}^{\mathrm{O}}\right] .
$$

Consider a special case where the control channels, i.e., the access and backhaul links, are modeled as orthogonal Rayleigh fading channels. Then, based on Equation $18, P_{n}^{S}$ in Equation 20 can be written as

$$
\begin{aligned}
P_{n}^{\mathrm{S}}= & {\left[1-\exp \left(\lambda^{\mathrm{D}}\left(d_{\mathrm{CU}, n}\right)\right)\right]+\exp \left(\lambda^{\mathrm{D}}\left(d_{\mathrm{CU}, n}\right)\right) } \\
& \times \prod_{n=1}^{N}\left[1-\exp \left(\lambda^{\mathrm{C}}\left(d_{\mathrm{CU}, n}\right)+\lambda^{\mathrm{O}}\left(d_{n, n}\right)\right)\right],
\end{aligned}
$$

where each link can be characterized with $\lambda(d)=-\frac{x}{\Omega(d)}$. Here, $d_{\mathrm{CU}, n}$ is the distance between TN $n$ and the $\mathrm{CU}$, and $d_{n, n}$ is the distance between UE $n$ and TN $n$.

\subsubsection{Coordinated scheduling}

In case of the CS scheme, it is possible that the TN will not transmit in the current resource block even if CSI sharing is not affected by the failure of control channels. This is because BPC is performed to control the interference, depending on the current channel conditions. Hence, TN $n$ will stay silent if

Case 1. The TN will not transmit in the current resource block, due to BPC, otherwise, 


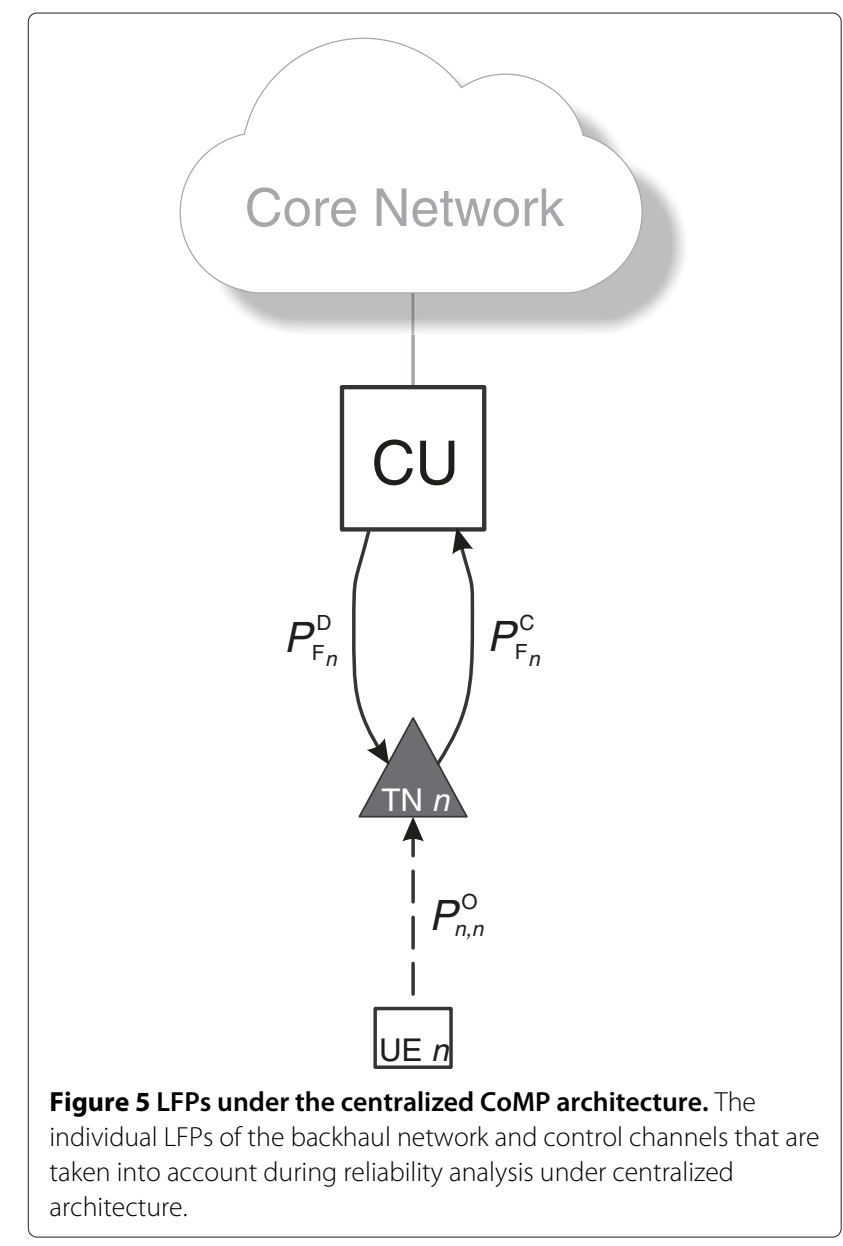

Case 2. The user data distributed from the CU to TN $n$ is lost. The probability that Case 1 happens is indicated as $P_{n}^{\mathrm{NS}}$, while Case 2 happens with probability $P_{\mathrm{F}_{n}}^{\mathrm{D}}$. Therefore, $P_{n}^{\mathrm{S}}$ for the CS scheme under centralized architecture can be expressed as

$$
P_{n}^{\mathrm{S}}=P_{\mathrm{F}_{n}}^{\mathrm{D}}+\left(1-P_{\mathrm{F}_{n}}^{\mathrm{D}}\right) P_{n}^{\mathrm{NS}} .
$$

Consider a special case where the control channels, i.e., the access and backhaul links, are modeled as Rayleigh fading channels; then, based on Equation $18, P_{n}^{\mathrm{S}}$ in Equation 22 can be written as:

$$
P_{n}^{\mathrm{S}}=\left[1-\exp \left(\lambda^{\mathrm{D}}\left(d_{\mathrm{CU}, n}\right)\right)\right]+\exp \left(\lambda^{\mathrm{D}}\left(d_{\mathrm{CU}, n}\right)\right) P_{n}^{\mathrm{NS}} .
$$

\subsection{Semi-distributed CoMP architecture}

\subsubsection{Coherent joint transmission}

In case of the JT scheme, TN $n$ will stay silent if all CSI vectors are lost at TN $n$. The probability that the local CSI vector $\left(\mathbf{h}_{n}\right)$ fed back by its own user, UE $n$, gets lost is $P_{n, n}^{\mathrm{O}}$, which is the LFP of the access link between UE $n$ and TN $n$. A non-local CSI ( $\mathbf{h}_{m}$ with $\left.m \neq n\right)$ can be lost at TN $n$ if $\mathbf{h}_{m}$ is not received at TN $m$ via access links from UE $m$ or $\mathbf{h}_{m}$ does not reach TN $n$ via backhaul links from TN $m$ (see Figure 6 for corresponding illustration). Hence, the probability that all non-local CSI vectors are lost at $\mathrm{TN} n$ is $\prod_{i=1, i \neq n}^{N}\left[P_{i, i}^{\mathrm{O}}+\left(1-P_{i, i}^{\mathrm{O}}\right) \cdot P_{\mathrm{F}_{n, i}}\right]$. Therefore, $P_{n}^{\mathrm{S}}$ for the JT scheme under semi-distributed architecture can be expressed as

$$
P_{n}^{\mathrm{S}}=P_{n, n}^{\mathrm{O}} \prod_{i=1, i \neq n}^{N}\left(P_{i, i}^{\mathrm{O}}+\left(1-P_{i, i}^{\mathrm{O}}\right) \cdot P_{\mathrm{F}_{n, i}}\right) .
$$

Consider a special case, where the control channels, i.e., the access and backhaul links, are modeled as Rayleigh fading channels without interference; then, based on Equation 18, $P_{n}^{S}$ in Equation 24 can be written as:

$$
\begin{aligned}
P_{n}^{\mathrm{S}}= & \left(1-\exp \left(\lambda^{\mathrm{O}}\left(d_{n, n}\right)\right)\right) \\
& \times \prod_{i=1, i \neq n}^{N}\left(1-\exp \left(\lambda^{\mathrm{O}}\left(d_{n, n}\right)+\lambda_{\mathrm{F}}\left(d_{n, i}\right)\right)\right) .
\end{aligned}
$$

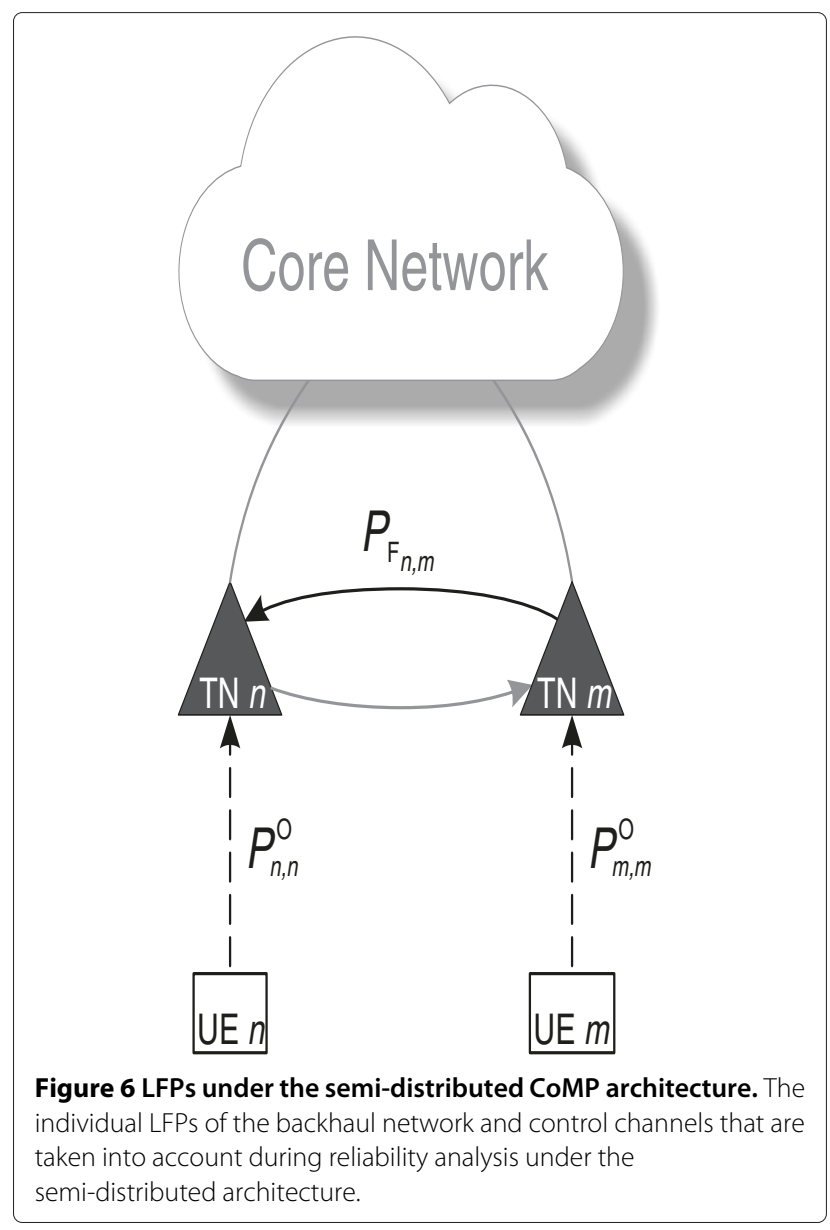




\subsubsection{Coordinated scheduling}

Considering the CS scheme under the semi-distributed architecture, $P_{n}^{\mathrm{NS}}$ will depend on the LFP of the backhaul links interconnecting the TNs and on the LFP of the access links. Thus $P_{n}^{\mathrm{S}}$ can be obtained as

$$
P_{n}^{\mathrm{S}}=P_{n}^{\mathrm{NS}} \text {. }
$$

\subsection{Fully distributed CoMP architecture 4.4.1 Coherent joint transmission}

In case of the JT scheme, $P_{n}^{\mathrm{S}}$ depends on whether CSI has reached TN $n$. Hence, TN $n$ will stay silent if all CSI vectors, sent from the UEs, are lost at TN $n$. All UEs broadcast their CSI, and the LFP of the access links between UE $m$ and TN $n$ is given by $P_{n, m}^{\mathrm{O}}$ (see Figure 7 for corresponding illustration). Therefore, $P_{n}^{\mathrm{S}}$ for the JT scheme under fully distributed architecture can be expressed as

$$
P_{n}^{\mathrm{S}}=\prod_{m=1}^{M} P_{n, m}^{\mathrm{O}}
$$

Consider a special case where the control channels, i.e., the access and backhaul links, are modeled as Rayleigh

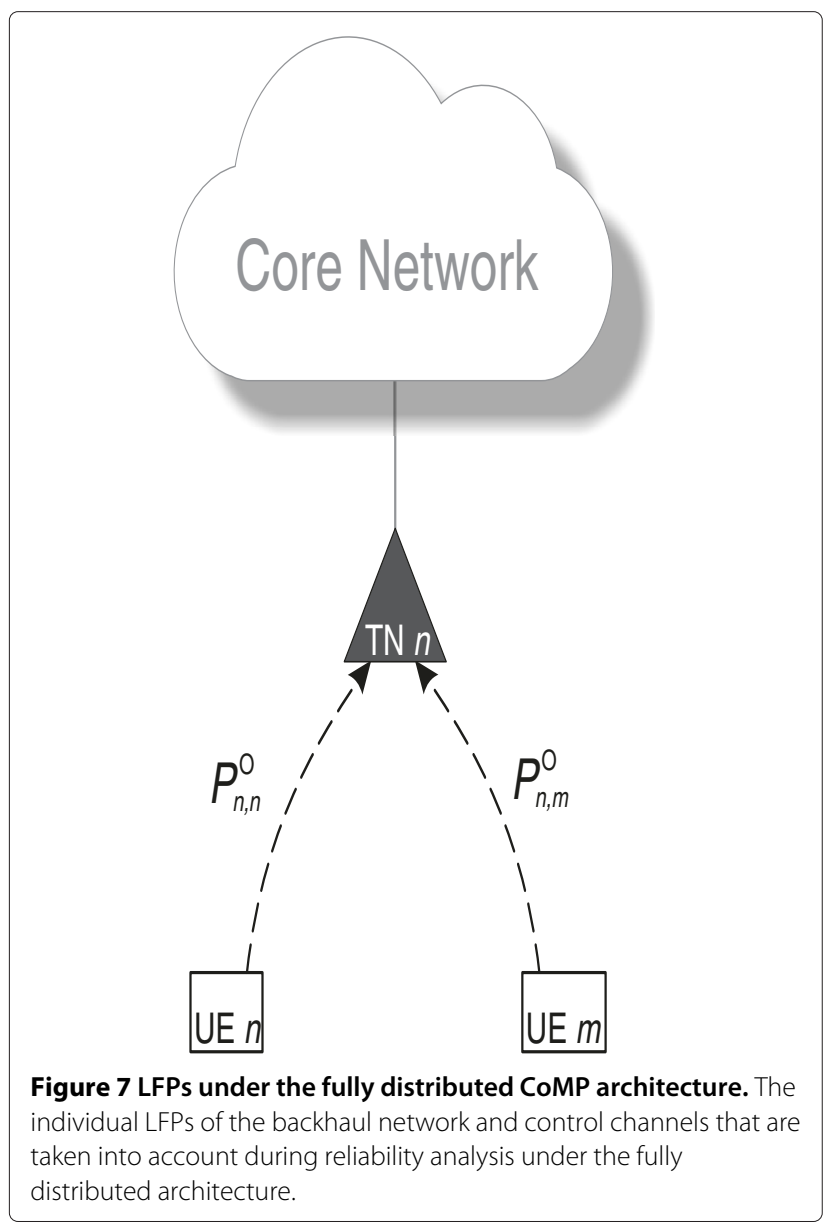

fading channels; then, based on Equation $18, P_{n}^{S}$ in Equation 27 can be written as:

$$
P_{n}^{\mathrm{S}}=\prod_{m=1}^{M}\left(1-\exp \left(\lambda^{\mathrm{O}}\left(d_{n, m}\right)\right)\right) .
$$

\subsubsection{Coordinated scheduling}

Considering the CS scheme under the fully distributed architecture, $P_{n}^{S}$ can be calculated using Equation 26. In this case, however, $P_{n}^{\mathrm{NS}}$ will depend on the LFPs of the access links between the UEs and TN $n$.

\section{Performance evaluation}

We consider the downlink of a CoMP cluster with $N=2$ and $N=3$ neighboring sectors respectively. For each cluster size, $N, M=N$ single-antenna UEs are grouped together using a particular resource slot. The cluster radius $R$ is $500 \mathrm{~m}$. The path loss model is $\mathrm{PL}(d)=128.1+$ $37.6 \log _{10}(d)$ in $\mathrm{dB}$, with $d$ given in $\mathrm{km}$. The system SNR is set to $18 \mathrm{~dB}$, which is defined as the received SNR at the boundary of the cell, assuming full power transmission $P_{\text {max }}$ from the TN, accounting only for path loss PL $(R)$ and ignoring fast fading [24]. The noise power, $\sigma^{2}$, is set to $-135 \mathrm{dBm}$.

To simplify our investigations, we assume that the UEs are collectively moving from their cell centers to the cluster center along the dashed line as depicted in Figure 8. SC transmission without TN coordination, denoted as single

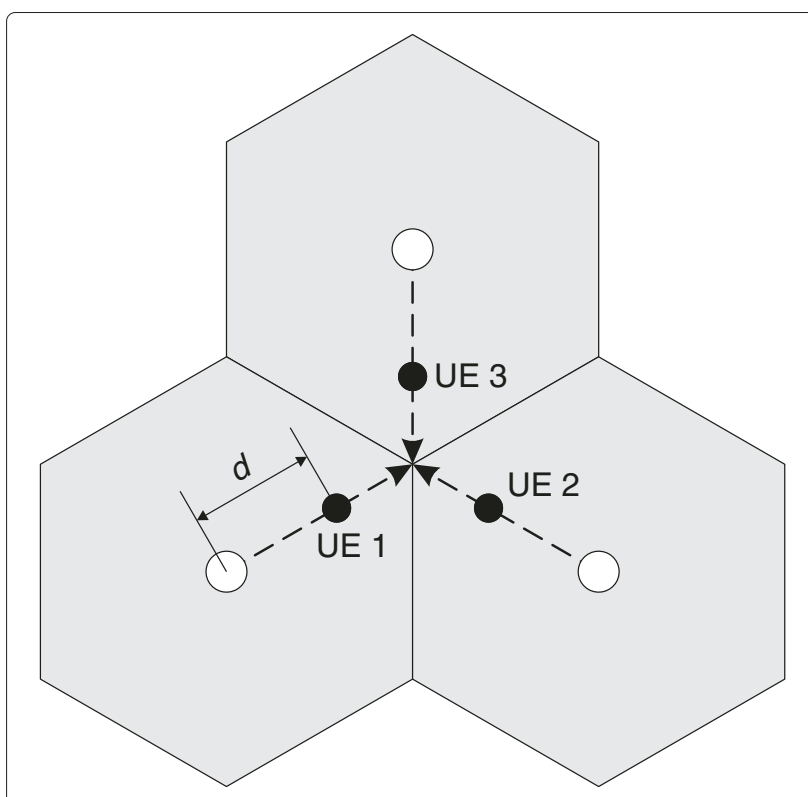

Figure 8 The simulated movement of the UEs, with cluster size $=3$, considered in numerical performance evaluation. As the UES approach the cluster center, the LFP between them and the TNs grows, impeding CSI feedback. Heightened interference also hampers data transmission. 
cell, is used as baseline. For each of the analyzed CoMP architectures, i.e., the centralized, semi-distributed, and fully distributed versions, the considered JT, CS, and SC transmission schemes are evaluated and compared. For each position, the average sum rate, $\bar{C}$, is obtained by averaging the sum rate of the cluster, obtained from Equation 5, over $10^{5}$ independent UE set realizations. The normalized distance of the UEs from the cell center is $\frac{d}{R}$.

\subsection{Average sum rate with perfect control information}

Figure $9 \mathrm{a}, \mathrm{b}$ plots the average sum rate of the cluster, $\bar{C}$, for different transmission schemes versus the normalized distance, with cluster size $N=2$ and $N=3$, respectively. In this case, all access and backhaul links are modeled to be completely reliable to illustrate how much performance gain is offered by the investigated CoMP schemes. Under such conditions, the architecture has no effect on the performance. The performance gain offered by the cooperative schemes is growing as interference becomes more severe when UEs are near the cluster center.

\subsection{Performance with unreliable control information \\ 5.2.1 Access link failure probability}

For both access and backhaul links, shadowing is ignored during the simulations ${ }^{\mathrm{e}}$. Only path loss and Rayleigh fading is considered; therefore, the signal power envelope is exponentially distributed at a given distance $d$. We assume that the UEs use orthogonal control channels during CSI feedback with a fixed transmit power. Consider the CSI feedback from UE $m$ to TN $n$ with distance $d$; the LFP of this access link can be evaluated by Equation 18, where the mean SNR can be obtained by [33]

$$
\Omega(d)=\frac{P_{\mathrm{t}}}{P L(d) \sigma^{2}} .
$$

We assume that the minimum required SNR for adequate reception, $x=\frac{P_{\mathrm{t}}}{P L\left(d_{\max }\right) \sigma^{2}}$. Since the control channels for CSI feedback are considered to be interferencefree $^{\mathrm{f}}$, the LFP of the access link between each UE $m$ and each $\mathrm{TN} n, P_{n, m}^{\mathrm{O}}$, in Equations 20 and 24 is independent of the cluster size, $N$. In Figure 10, the LFP of the access link between a certain UE and its serving $\mathrm{TN}, P_{n, n}^{\mathrm{O}}$, is plotted versus the normalized distance $\frac{d}{R}$.

\subsubsection{The probability of a TN staying silent}

For a symmetric CoMP cluster shown in Figure 8, all backhaul links are assumed to have the same LFP, $P_{\mathrm{F}}$. Since the UEs are moving collectively, all access links between the UEs and their serving TNs are characterized with the same outage probability, $P^{\mathrm{O}}$, and all access channels between the UEs and their neighboring TNs are characterized with the same outage probability, $P_{n, m}^{\mathrm{O}}$. Note that $P^{\mathrm{O}}$ and $P_{n, m}^{\mathrm{O}}$ are functions of distance $d$, being the same value for all UEs in each realization. In this case, the Equations 20, 22, 24, and 27 are reduced to the following forms. For JT under the centralized architecture:

$$
P_{n}^{\mathrm{S}}=P_{\mathrm{F}}+\left(1-P_{\mathrm{F}}\right)\left[P_{\mathrm{F}}+\left(1-P_{\mathrm{F}}\right) P^{\mathrm{O}}\right]^{N}
$$

a)

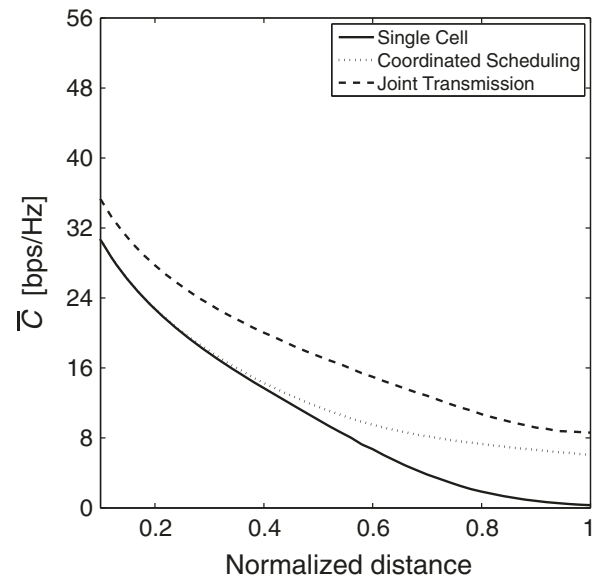

b)

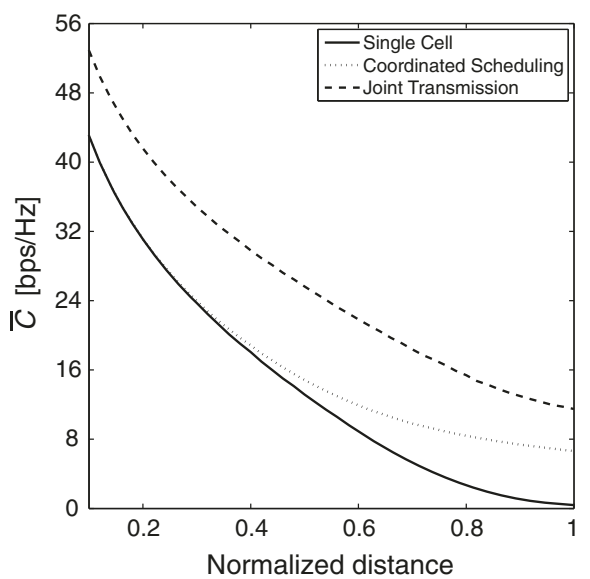

Figure 9 The average sum rate of the cluster, $\overline{\boldsymbol{C}}$ vs. normalized distance, $\frac{\boldsymbol{d}}{R}$. Values are plotted for (a) cluster size $=2$, and (b) cluster size $=3$. It is assumed that the control channels are completely reliable, i.e., the LFP of all access links, $P_{n, m}^{O}$, and the LFP of all backhaul links, $P_{F_{n, m}}$, is set to 0 . 


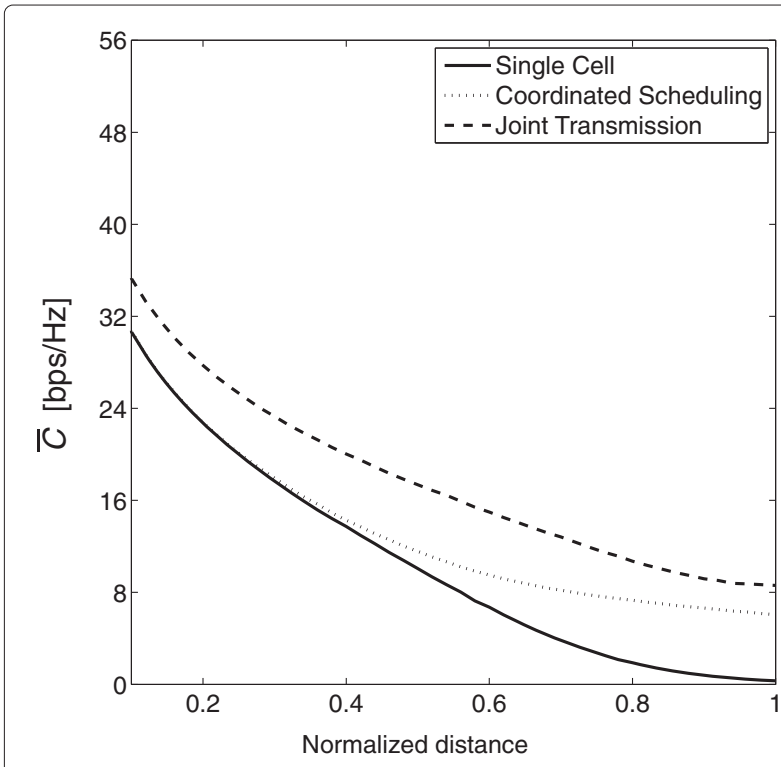

Figure 10 The LFP of the control channel over the access link used for CSI feedback. The LFP, $P_{\text {out }}$, is plotted vs. the normalized distance, $\frac{d}{R}$, between UE $n$ and its serving TN $n$. It is assumed that the control channels are orthogonal for different users.

For CS under the centralized architecture:

$$
P_{n}^{\mathrm{S}}=P_{\mathrm{F}}+\left(1-P_{\mathrm{F}}\right) P_{n}^{\mathrm{NS}}
$$

For JT under the semi-distributed architecture:

$$
P_{n}^{\mathrm{S}}=P^{\mathrm{O}}\left(P^{\mathrm{O}}+\left(1-P^{\mathrm{O}}\right) P_{\mathrm{F}}\right)^{N-1}
$$

For JT under the fully distributed architecture:

$$
P_{n}^{\mathrm{S}}=\prod_{m=1}^{M} P_{n, m}^{\mathrm{O}} .
$$

If we consider the special case of modeling the wireless links with Rayleigh fading, Equations 30 to 33 will have the following form:

$$
\begin{aligned}
P_{n}^{\mathrm{S}}= & {\left[1-\exp \left(\lambda_{\mathrm{F}}\left(d_{\mathrm{CU}, n}\right)\right)\right]+\exp \left(\lambda_{\mathrm{F}}\left(d_{\mathrm{CU}, n}\right)\right) } \\
& \times\left[1-\exp \left(\lambda_{\mathrm{F}}\left(d_{\mathrm{CU}, n}\right)+\lambda^{\mathrm{O}}\left(d_{n, n}\right)\right)\right]^{N}, \\
P_{n}^{\mathrm{S}}= & {\left[1-\exp \left(\lambda_{\mathrm{F}}\left(d_{\mathrm{CU}, n}\right)\right)\right]+\exp \left(\lambda_{\mathrm{F}}\left(d_{\mathrm{CU}, n}\right)\right) P_{n}^{\mathrm{NS}}, }
\end{aligned}
$$

$$
\begin{aligned}
P_{n}^{\mathrm{S}}= & \left(1-\exp \left(\lambda^{\mathrm{O}}\left(d_{n, n}\right)\right)\right)\left[\left(1-\exp \left(\lambda^{\mathrm{O}}\left(d_{n, n}\right)\right)\right)\right. \\
& \left.+\exp \left(\lambda^{\mathrm{O}}\left(d_{n, n}\right)\right)\left(1-\exp \left(\lambda_{\mathrm{F}}\left(d_{\mathrm{CU}, n}\right)\right)\right)\right]^{N-1} \\
P_{n}^{\mathrm{S}}= & \prod_{m=1}^{M}\left(1-\exp \left(\lambda^{\mathrm{O}}\left(d_{n, m}\right)\right)\right) .
\end{aligned}
$$

Note that $P^{\mathrm{S}}$ does not directly limit $\bar{C}$ since the silence of a TN also decreases the inter-cell interference in the neighboring cells. Figure 11 shows the probability of a certain TN $n$ staying silent in a resource slot, $P_{n}^{\mathrm{S}}$, versus the normalized distance, for each transmission scheme and system architecture. The backhaul LFP, $P_{\mathrm{F}}$, is set to 0.1 .

We can see that in the region where the UEs are close to their serving TNs, a certain TN will stay silent with a significantly higher probability under the centralized architecture with both JT and CS schemes. From Equations 30 to 33, it can be seen that $P_{n}^{S}$ for the JT scheme under centralized architecture is dominated by backhaul LFP, $P_{F}$, while the impact of backhaul LFP on $P_{n}^{S}$ is much less under the semi-distributed architecture. Backhaul LFP has no impact on the performance under the fully-distributed architecture.

For the JT scheme under the semi-centralized architecture, $P^{\mathrm{S}}$ shows a similar trend as with $\mathrm{SC}$ transmission. However, under the fully distributed architecture, $P^{\mathrm{S}}$ drops after an initial rise as the UEs get closer to the cluster center. This is due to the fact that the UEs use orthogonal access links and the broadcasted CSI vectors reach the neighboring TNs with a higher probability since the LFP of the access links is a function of their access distance. For the JT scheme, $P^{\mathrm{S}}$ appears to be inversely proportional to the cluster size under all architectures.

For the CS scheme, $P^{\mathrm{S}}$ reaches the highest values under the fully distributed architecture as the UEs get closer to the cluster center. This is due to the fact that the BPC algorithm reduces interference by keeping more TNs silent on average. For the CS scheme, $P^{\mathrm{S}}$ appears to be directly proportional to the cluster size under all architectures.

\subsubsection{Average sum rate evaluation}

Figure 12 plots the average sum rate of the cluster, $\bar{C}$, for different transmission schemes versus the normalized distance with cluster size $N=3$. It is assumed that all backhaul links have the same LFP, $P_{\mathrm{F}}=0.1$. As shown in Figure 10, under the assumption of fixed transmit power at each UE, the LFP of the access links between the UEs and the TNs is a function of distance $d$. Compared to the case where all control channels are assumed to be fully reliable (Figure 9), the performance of all CoMP 
a)

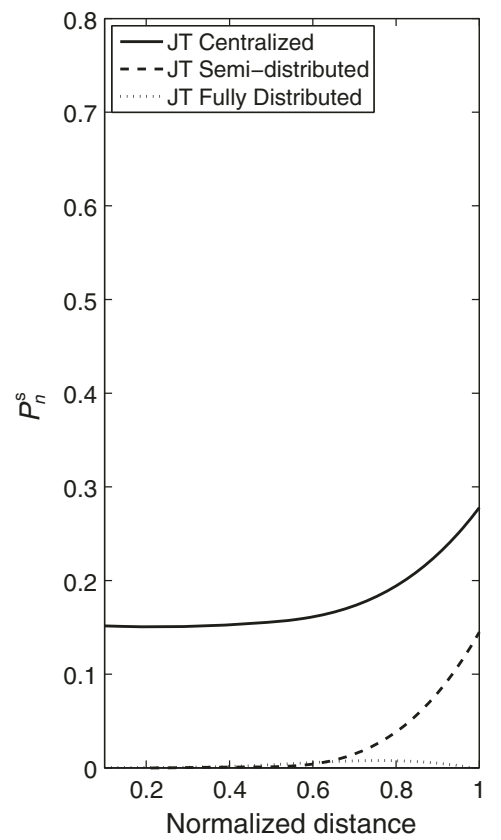

b)

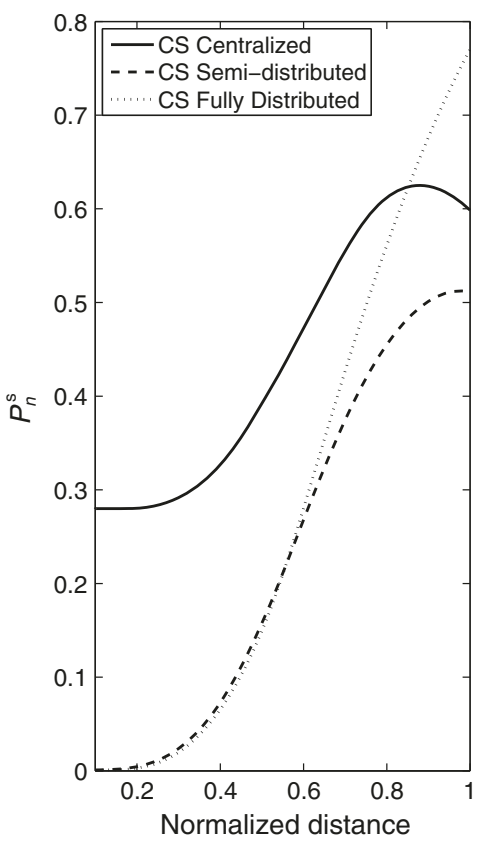

Figure 11 The probability of a certain TN $\boldsymbol{n}$ staying silent, $P_{n}^{S}$, vs. normalized distance, $\frac{d}{R}$. Values plotted for (a) JT scheme, and (b) CS scheme. Cluster size $=3$, and the UEs advance toward the cluster center. The LFP of the backhaul links, $P_{F}$, is set to 0.1 .

a)

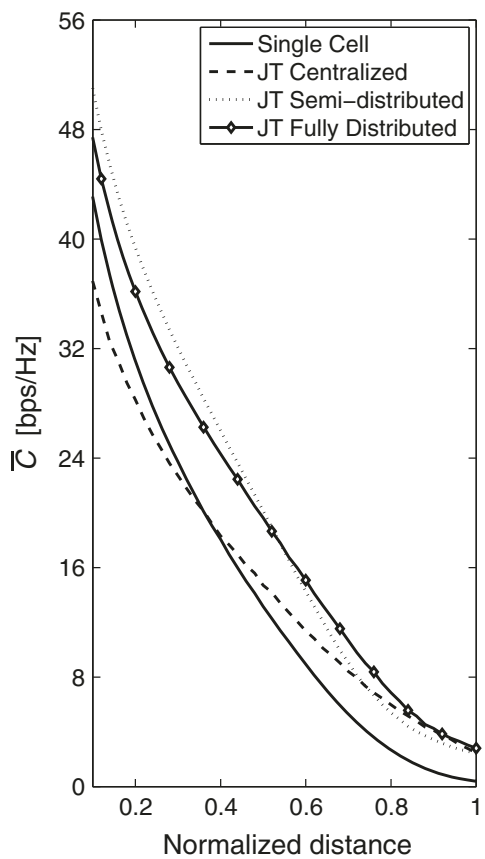

b)

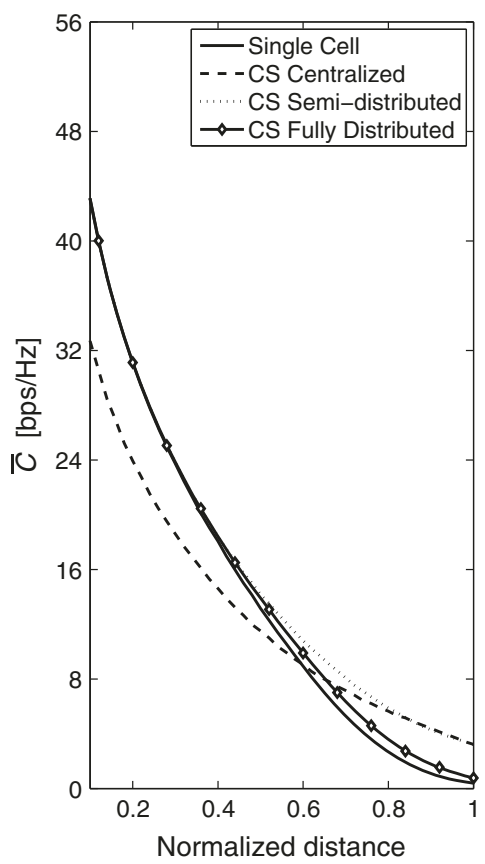

Figure 12 The average sum rate of the cluster, $\overline{\boldsymbol{C}}$, vs. normalized distance, $\frac{\boldsymbol{d}}{R}$. Values are plotted for (a) JT scheme, and (b) CS scheme. Cluster size $=3$, and the UEs advance toward the cluster center. The LFP of the backhaul links, $P_{F}$, is set to 0.1 . 
schemes declines. It is apparent, however, that the control channel architecture of the cluster has a significant effect on the average sum rate. Under the centralized architecture, the performance of the CoMP schemes is worse than SC transmission since the backhaul link between the TNs and the CU is prone to errors, even when the UEs are close to their serving TN. As the UEs move toward the cluster center, the path loss over the access link and the interference gets larger, thus the performance of SC transmission drops sharply. Initially, the JT scheme under semi-distributed architecture offers the highest achievable sum rate; however, this gain diminishes quickly as control channel unreliability over the access link grows. As the UEs get closer to the cluster center, the distance between the UEs and the neighboring TNs gets smaller. In this region, the JT scheme offers the best performance under the fully distributed architecture because CSI is shared over the access links. However, the CS scheme does not perform well near the cluster center under the fully distributed architecture because each TN decides not to transmit with high probability.

Figure 13 plots $\bar{C}$ against varying backhaul LFP, $P_{\mathrm{F}}$, when the normalized distance of the UEs from the cell centers is 0.4. The performance of the $\mathrm{SC}$ transmission and the fully distributed architecture does not depend on $P_{\mathrm{F}}$ since the control signals are only transmitted through the access links. The centralized architecture is the most sensitive to backhaul LFP, and if the backhaul links fail with certainty, no transmission is possible. The performance of the semi-distributed architecture converges to SC transmission because if the gathered system channel matrix at TN $n$ only contains information from UE $n$ with high probability, the application of precoding and scheduling algorithms becomes redundant.

\section{Conclusions}

In this paper, the effects of control channel reliability on the performance of a cluster of cooperative transmission nodes has been studied. In particular, two transmission schemes, coherent joint transmission and coordinated scheduling, were evaluated under the centralized, semidistributed, and fully distributed CoMP architectures. The scenarios were assessed in terms of average sum rate of the cluster, and traditional single cell transmission served as a baseline for comparison. General expressions were derived to show how unreliable backhaul links and unreliable access links affect quality of service. Numerical results show that cooperative transmission techniques have the potential to improve the performance of the cellular system, in terms of sum rate. However, the performance of the system highly depends on the reliability of the control channels and, more importantly, on the probability of successful channel state information exchange. Although all examined scenarios suffer from performance a)

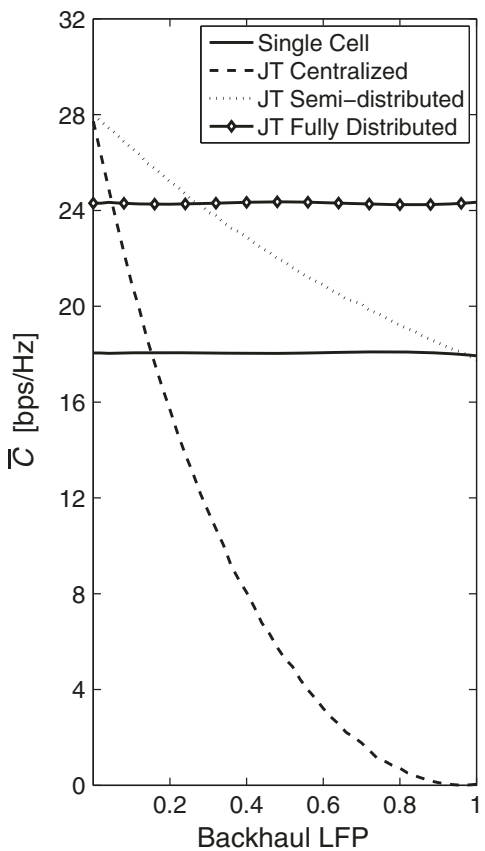

b)

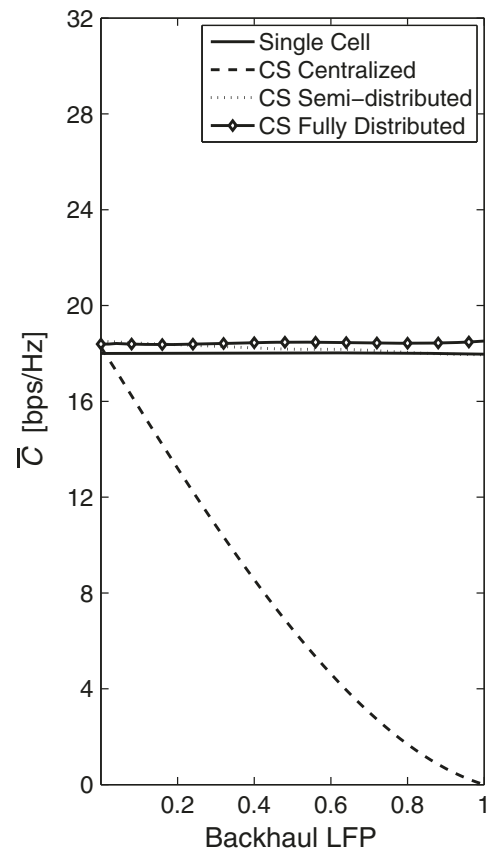

Figure 13 The average sum rate of the cluster, $\overline{\mathbf{C}}$ vs. backhaul LFP, $\boldsymbol{P}_{\mathbf{F}}$. Values are plotted for (a) JT scheme, and (b) CS scheme. Cluster size $=3$, and the normalized distance, $\frac{d}{R}$, of the UEs from the cell centers is 0.4 . 
degradation as intra-cell interference increases, the coherent joint transmission scheme proved to be more robust under the fully distributed architecture. With the coherent joint transmission scheme also higher sum rates can be achieved. The semi-distributed and fully distributed architectures are less sensitive to backhaul unreliability; however, they require all cooperating TNs to be capable of performing precoding or making scheduling decisions. In this paper, explicit channel state information is considered as the feedback information. In practice, the control information might be a precoding matrix index that corresponds to the preferred codebook or the quantized version of the channel state information. The masking concept is still valid for this case and will be considered in our future work. In this work the authors were focused on better understanding of how the performance of a cluster of low-power cells with similar output power can be evaluated and improved. A realistic HetNet would comprise of a multitude of such clusters and of other types of access nodes with different output powers. To maintain simplicity, a small cluster was studied and the effects of adjacent clusters and cross-tier interference is not considered in this article, but shall be part of any future work.

\section{Endnotes}

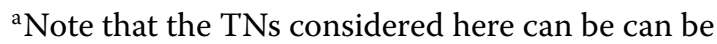
represented as a set of base stations, relay nodes, femtocells, or users or a combination of different kinds of access nodes.

${ }^{\mathrm{b}}$ To include the channel estimation error in the proposed model, $\mathbf{H}$ can be replaced with $\mathbf{H}+\mathbf{N}$ in Equation 9, where $\mathbf{N}$ denotes the channel estimation error matrix.

${ }^{c}$ Note that if different channel feedback mechanisms are considered, there exists cases where only individual elements of the local gathered channel system matrix are lost. In this paper, however, we assume that under the distributed scenario, each UE $m$ will jointly encode the local CSI vector, $\mathbf{h}_{m}$, and then broadcast it back to all TNs. Therefore, if the access link between the UE $m$ and a certain TN $n$ is in outage, the whole channel vector, $\mathbf{h}_{m}$, will get lost.

${ }^{\mathrm{d}}$ Here, we do not include shadow fading for analytical tractability, but it is straightforward to extend the modeling by resorting to numerical methods. Shadow fading models for dense small cell networks is an important open research problem.

${ }^{\mathrm{e}}$ Note that shadowing can be introduced to our model by adding a shadowing factor to each of the $\Omega(\mathrm{d})$ values in Equation 29.

${ }^{\mathrm{f}}$ Note that the data channel considered in this paper is not interference-free since the group of users sharing the same resource slot suffer from inter-cell interference.

\section{Competing interests}

The authors declare that they have no competing interests.

\section{Acknowledgements}

This work is supported by the Swedish Agency for Innovation Systems (VINNOVA) within the P36604-1 MAGIC project and the Swedish Research Council VR under the project 621-2009-4555 Dynamic Multipoint Wireless Transmission. Part of this work has been performed in the framework of the FP7 project ICT-317669 METIS, which is partly funded by the European Union. The authors would like to acknowledge the contributions of their colleagues in METIS, although the views expressed are those of the authors and do not necessarily represent the project.

Received: 19 February 2013 Accepted: 19 January 2014

Published: 20 February 2014

\section{References}

1. D Mcqueen, The momentum behind LTE adoption. Commun. Mag., IEEE. 47(2), 44-45 (2009)

2. C Gandarillas, V Iglesias, M Aparicio, Mino-E Diaz, P Olmos, A new approach for improving indoor LTE coverage, in GLOBECOM Workshops (GCWkshps): 5-9 Dec 2011; Houston (IEEE, Piscataway, 2011), pp. 1330-1335

3. V Chandrasekhar, J Andrews, A Gatherer, Femtocell networks: A survey. Commun. Mag, IEEE. 46(9), 59-67 (2008)

4. A Ghosh, N Mangalvedhe, R Ratasuk, B Mondal, M Cudak, E Visotsky, T Thomas, J Andrews, P Xia, HS Jo, H Dhillon, T Novlan, Heterogeneous cellular networks: From theory to practice. Commun. Mag., IEEE. 50(6), 54-64 (2012)

5. G Boudreau, J Panicker, N Guo, R Chang, N Wang, S Vrzic, Interference coordination and cancellation for $4 \mathrm{G}$ networks. Commun. Mag, IEEE. 47(4), 74-81 (2009)

6. A Barbieri, P Gaal, S Geirhofer, T Ji, D Malladi, Y Wei, F Xue, Coordinated downlink multi-point communications in heterogeneous cellular networks, in Information Theory and Applications Workshop (ITA): 5-10 Feb 2012; San Diego (IEEE, Piscataway, 2012), pp. 7-16

7. M Sawahashi, Y Kishiyama, A Morimoto, D Nishikawa, M Tanno, Coordinated multipoint transmission/reception techniques for LTE-advanced [Coordinated and Distributed MIMO]. Wireless Commun., IEEE. 17(3), 26-34 (2010)

8. D Gesbert, S Hanly, H Huang, S Shamai Shitz, O Simeone, W Yu, Multi-cell MIMO cooperative networks: A new look at interference. Selected Areas in Communications, IEEE J. 28(9), 1380-1408 (2010)

9. D Lee, H Seo, B Clerckx, E Hardouin, D Mazzarese, S Nagata, K Sayana, Coordinated multipoint transmission and reception in LTE-advanced: deployment scenarios and operational challenges. Commun. Mag., IEEE. 50(2), 148-155

10. S Ramprashad, H Papadopoulos, A Benjebbour, Y Kishiyama, N Jindal, G Caire, Cooperative cellular networks using multi-user MIMO: Trade-offs, overheads, and interference control across architectures. Communications Magazine, IEEE. 49(5), 70-77 (2011)

11. A Papadogiannis, H Bang, D Gesbert, E Hardouin, Efficient selective feedback design for multicell cooperative Networks. Vehicular Technology, IEEE Trans. 60, 196-205 (2011)

12. P Marsch, GP Fettweis, Coordinated Multi-Point in Mobile Communications: from theory to practice (Cambridge University Press, Cambridge, 2011)

13. O Simeone, O Somekh, E Erkip, H Poor, S Shamai, Robust communication via decentralized processing with unreliable backhaul links. Information Theory, IEEE Trans. 57(7), 4187-4201 (2011)

14. A Papadogiannis, T Svensson, Performance analysis of centralized relay selection with unreliable control information, in Vehicular Technology Conference (VTC Fall): 3-6 Sept 2012; Quebec (IEEE, Piscataway, 2012), pp. 1-5

15. B Maham, A Behnad, M Debbah, Analysis of outage probability and throughput for half-duplex hybrid-ARQ relay channels. Vehicular Technology, IEEE Trans. 61(7), 3061-3070 (2012)

16. F Pantisano, M Bennis, W Saad, M Debbah, M Latva-aho, On the impact of heterogeneous backhauls on coordinated multipoint transmission in femtocell networks, in IEEE International Conference on Communications (ICC): 10-15 Jun 2012; Ottawa (IEEE, Piscataway, 2012), pp. 5064-5069

17. Z Mayer, J Li, A Papadogiannis, T Svensson, On the impact of backhaul channel reliability on cooperative wireless networks, in IEEE International 
Conference on Communications: 9-13 Jun 2013; Budapest (IEEE, Piscataway, 2013), pp. 5284-5289

18. P de Kerret, D Gesbert, Degrees of freedom of the network MIMO channel with distributed CSI. Information Theory, IEEE Trans. 58(11), 6806-6824 (2012)

19. D Villa, C Castellanos, I Kovacs, F Frederiksen, K Pedersen, Performance of downlink UTRAN LTE under control channel constraints, in Vehicular Technology Conference: 11-14 May 2008; Singapore (IEEE, Piscataway, 2008), pp. 2512-2516

20. L Zhang, Z He, K Niu, X Wang, P Skov, Impact of control channel constraints on downlink performance comparison of MIMO transmission schemes in 3GPP LTE, in IEEE 20th International Symposium on Personal, Indoor and Mobile Radio Communications: 13-16 Sept 2009; Tokyo (IEEE, Piscataway, 2009), pp. 2075-2079

21. J Li, T Eriksson, T Svensson, C Botella, Power allocation for two-cell two-user joint transmission. Commun. Lett, IEEE. 16(9), 1474-1477 (2012)

22. O Tipmongkolsilp, S Zaghloul, A Jukan, The evolution of cellular backhaul technologies: current issues and future trends. Commun. Surv. Tutorials, IEEE. 13, 97-113 (2011)

23. M Coldrey, H Koorapaty, J Berg, Z Ghebretensae, J Hansryd, A Derneryd, $S$ Falahati, Small-cell wireless backhauling: a non-line-of-sight approach for point-to-point microwave Links, in Vehicular Technology Conference (VTC Fall): 3-6 Sept 2012; Quebec (IEEE, Piscataway, 2012), pp. 1-5

24. J Li, A Papadogiannis, R Apelfrojd, T Svensson, M Sternad, Performance evaluation of coordinated multi-point transmission schemes with predicted CSI, in IEEE 23rd International Symposium on Personal Indoor and Mobile Radio Communications (PIMRC): 9-12 Sept 2012; Sydney (IEEE, Piscataway, 2012), pp. 1055-1060

25. A Papadogiannis, E Hardouin, D Gesbert, Decentralising multicell cooperative processing: A novel robust framework. EURASIP J. Wirel. Commun. Netw. 2009, 4:1-4:10 (2009). http://dx.doi.org/10.1155/2009/ 890685

26. A Gjendemsjo, D Gesbert, G Oien, S Kiani, Binary power control for sum rate maximization over multiple interfering links. Wireless Commun., IEEE Trans. 7(8), 3164-3173 (2008)

27. J Li, T Svensson, C Botella, T Eriksson, $X X \mathbf{X}, X$ Chen, Joint scheduling and power control in coordinated multi-point clusters, in Vehicular Technology Conference (VTC Fall): 5-8 Sept 2011; San Francisco (IEEE, Piscataway, 2011), pp. 1-5

28. H Zhang, H Dai, Cochannel interference mitigation and cooperative processing in downlink multicell multiuser MIMO networks. EURASIP J. Wirel. Commun. Netw. 2004(2), 222-235 (2004). http://dx.doi.org/10. $1155 / S 1687147204406148$

29. V Jungnickel, L Thiele, T Wirth, T Haustein, S Schiffermuller, A Forck, S Wahls, S Jaeckel, S Schubert, H Gabler, C Juchems, F Luhn, R Zavrtak, H Droste, G Kadel, W Kreher, J Mueller, W Stoermer, G Wannemacher, Coordinated multipoint trials in the downlink, in GLOBECOM Workshops: 30 Nov-4 Dec 2009; Honolulu (IEEE Piscataway, 2009), pp. 1-7

30. L Scalia, T Biermann, C Choi, K Kozu, W Kellerer, Power-efficient mobile backhaul design for CoMP support in future wireless access systems, in IEEE Conference on Computer Communications Workshops (INFOCOM WKSHPS): 10-15 Apr 2011; Shanghai (IEEE, Piscataway, 2011), pp. 253-258

31. R Fritzsche, G Fettweis, CSI distribution for joint processing in cooperative cellular networks, in IEEE Vehicular Technology Conference (VTC Fall):5-8 Sept 2011; San Francisco (IEEE, Piscataway, 2011), pp. 1-5

32. A Goldsmith, Wireless Communications (Cambridge University Press, Cambridge, 2005)

33. KW Sowerby, Outage probability in mobile radio systems. PhD thesis, The University of Auckland, 1989

doi:10.1186/1687-1499-2014-28

Cite this article as: Mayer et al.: On the impact of control channel reliability on coordinated multi-point transmission. EURASIP Journal on Wireless Communications and Networking 2014 2014:28.

\section{Submit your manuscript to a SpringerOpen ${ }^{\circ}$ journal and benefit from:}

- Convenient online submission

- Rigorous peer review

- Immediate publication on acceptance

- Open access: articles freely available online

- High visibility within the field

- Retaining the copyright to your article

Submit your next manuscript at $\gg$ springeropen.com 